\title{
Pattern separation and goal-directed behavior in the aged canine
}

\author{
Shikha Snigdha, ${ }^{1}$ Michael A. Yassa, ${ }^{1,2,3}$ Christina deRivera, ${ }^{4}$ Norton W. Milgram, ${ }^{4}$ \\ and Carl W. Cotman ${ }^{1,2,3}$
}

${ }^{1}$ UC Institute for Memory Impairments and Neurological Disorders (UCI MIND), University of California, Irvine, California 92697, USA;
${ }^{2}$ Department of Neurobiology and Behavior, University of California, Irvine, California 92697, USA; ${ }^{3}$ Department of Neurology, School
of Medicine, University of California, Irvine, California 92697, USA; ${ }^{4}$ CanCog Technologies Inc., Toronto, Ontario M5A 4K2, Canada

The pattern separation task has recently emerged as a behavioral model of hippocampus function and has been used in several pharmaceutical trials. The canine is a useful model to evaluate a multitude of hippocampal-dependent cognitive tasks that parallel those in humans. Thus, this study was designed to evaluate the suitability of pattern separation task(s) for detecting age-related changes in canines. We also assessed the dogs' ability to show pattern separation and discrimination reversal, which provides a novel extension of the pattern separation learning literature. Our data show that aged dogs are impaired on a complex pattern separation task (six-well task) relative to easier tasks (four-well or six-well pattern discrimination task), and that the age-related deficits are due to loss of perceptual and inhibitory control in addition to the loss of spatial discrimination and pattern separation ability. Our data also suggest that aged animals show pattern separation deficits when the objects are brought progressively closer together while changing the location of both correct and incorrect objects. However, if the location of any one object is fixed the animals tend to use alternate strategies. Overall, these data provide important insight into age-related pattern separation deficits in a higher animal model and offers additional means for evaluating the impact of lifestyle and pharmaceutical interventions on episodic memory in preclinical trials.

There is a need to develop behavioral tests in higher animal models, which reflect the complexity of those human cognitive functions that are most sensitive to age-related cognitive decline. In this regard, the dog is particularly promising. The use of dogs in aging studies provides some unique advantages, as dogs are easy to handle and often share a common environment (including diet) with humans. In addition, both in cognitive capability, where they are able to learn a multitude of tasks (Head et al. 2008), and in the development of age-related brain pathology (Cotman and Head 2008) canines can parallel humans. Specifically, dogs capture the phenotype of early AD neuropathology by developing $\mathrm{A} \beta$ plaques and showing neuronal and synaptic loss with advancing age. Finally, dogs offer predictive validity to studies when translating results to human clinical trials, as they absorb pharmaceuticals with similar if not identical pharmacokinetics.

The canine has proven to be especially useful for pharmaceutical studies and for evaluating the role of behavioral lifestyles on age-related cognitive decline. Over the past few years, pattern separation tasks assessing the ability to discriminate among similar objects or spatial locations have provided a valuable approach for evaluating various pharmaceutical trials in humans (Bakker et al. 2012). Pattern separation is defined as the ability to discriminate among similar experiences and is a critical feature of episodic memory (Yassa et al. 2011b; Yassa and Stark 2011). Studies have shown that small changes in the environment in which rats explore can significantly alter the activity patterns of placemodulated granule cells and that these changes can enable the hippocampus to delineate the different memories of similar events (Goodrich-Hunsaker et al. 2008). Marrone et al. (2011) provided some of the first evidence of how age-related changes in the

\section{Corresponding author: ssnigdha@uci.edu}

Article is online at http://www.learnmem.org/cgi/doi/10.1101//m.043422.116. dentate gyrus in rodents may affect pattern separation and spatial memory. Using a marker of cellular activity (zif268/egr1) to examine granule cell activity in young and older animals, the authors showed that aged animals recruited distinct granule cell populations in their visits to similar or same environments. The authors reported that age-related changes in pattern separation correlated with a decreased ability of older animals to disambiguate similar contexts when performing a sequential spatial recognition task (Marrone et al. 2011).

A behavioral task suitable for detecting age-related changes in pattern separation in canines would thus be an important addition for both pharmaceutical and nonpharmaceutical studies for evaluating the impact of lifestyle and therapeutic interventions on episodic memory.

There has been considerable recent interest in mechanisms involved in pattern separation in the context of theories of hippocampal function (Rolls 2015), particularly in aging (Bennett et al. 2015). And while the role of hippocampus in pattern separation is well accepted, it is not clear whether other neurocognitive mechanisms may compensate for the loss of this particular aspect of cognition. In fact, one limitation in this area of research (particularly for preclinical testing) has been the use of tests that use relatively general measures of learning and memory including spatial learning, recollection, and recognition. It is entirely possible that using alternative strategies, such as allocentric or egocentric strategies, relative familiarity strength, or even executive function and increasing repetition of events, may alter the degree of observed memory impairment in young versus aged animals (Aimone

(C) 2017 Snigdha et al. This article is distributed exclusively by Cold Spring Harbor Laboratory Press for the first 12 months after the full-issue publication date (see http://learnmem.cshlp.org/site/misc/terms.xhtml). After 12 months, it is available under a Creative Commons License (AttributionNonCommercial 4.0 International), as described at http://creativecommons. org/licenses/by-nc/4.0/. 
et al. 2011; Rich and Shapiro 2009). Thus development of a task that examines these components separately would be of significant benefit.

In this study, our goal is to develop a pattern separation task that could be used in dogs and to examine how performance on the task was affected by age. We initially based the protocols on tasks described by Reagh et al. (2014) in humans and Bussey et al. (2001) in mice to evaluate pattern separation learning. We specifically focused on three distinct tasks, the first involved a four-well test paradigm and the next two strategies utilized six-well paradigms. Specifically, the first task assessed responding to one of two identical target objects placed at two of four possible positions (food wells). The distance between the objects varied, from close, to medium to far apart and we initially only moved the location of the correct object. The "incorrect object" remained always to one side of the test apparatus. The second task (six-well paradigm) used three different spatial configurations and objects could be placed at six possible positions (food wells). In this case, the position of both the correct and "incorrect" objects were varied across trials and could be to the right or left of the animal. Furthermore, in order to eliminate a corner effect (which may enable the use of alternate strategies such as the use of an allocentric strategy by using an external landmark to reach the correct response), no objects were placed in corner locations during test. Finally, in another six-well paradigm (task 3), we used the corners but either had the correct object on the same side of the animals (problem set 1), while progressively decreasing separation of objects or used the corners but switched the correct object from left to right (problem set 2 ) while decreasing separation of objects progressively. This task combined relevant aspects of the first two tasks in (1) using the corners, (2) moving both correct and incorrect objects, and (3) placing correct object on the same side for one half of the tests and switching this around for the second half.

This design allowed us to distinguish among the use a simple strategy subjects learn to keep away from the fixed incorrect position versus egocentric strategies, in which the correct response could be based on self-reference, such as left and right (Milgram et al. 2002), versus allocentric strategies where subjects used the corners as an external reference points to reach the correct response, or finally a pattern separation strategy in which interference between overlapping spatial information is minimized. Last, we also added pattern separation reversal tasks, which address an issue that prior behavioral work assessing pattern separation has not evaluated.

\section{Results}

\section{Four-well pattern separation test}

The four-well pattern separation task (Fig. 1) was based on traditional pattern separation tasks with distance between objects being the only factor that changed for each problem set. However, we found no significant effect of spatial pattern and only a marginal effect of age on this task. A two-way ANOVA on the learning phase scores confirmed this (interaction effect; $F_{(2,28)}=0.32$, $P=0.76$, main effect of age; $F_{(1,14)}=4, P=0.06$, main effect of pattern; $F_{(2,28)}=2.12, P=0.13$, Fig. $\left.2 \mathrm{~A}\right)$.

There was also no effect of age or spatial pattern in the first reversal (interaction effect; $F_{(2,28)}=0.15, P=0.85$, main effect of age; $F_{(1,14)}=0.03, P=0.87$, main effect of pattern; $F_{(2,28)}=1.55$, $P=0.22$, Fig. $2 \mathrm{~B}$ ) or second reversal (interaction effect; $F_{(2,28)}=$ $1.69, P=0.20$, main effect of age; $F_{(1,14)}=2.26, P=0.17$, main effect of pattern; $F_{(2,28)}=0.03, P=0.79$, Fig. 2C).

In addition we did not observe any age- or task-related difference in the latency of response during learning (interaction effect; $F_{(2,28)}=0.42, P=0.66$, main effect of age; $F_{(1,14)}=0.96, P=0.32$, main effect of pattern; $F_{(2,28)}=0.12, P=0.88$, Fig. $\left.3 \mathrm{~A}\right)$. During the first reversal there was no effect of interaction or age (interaction effect; $F_{(2,28)}=1.32, P=0.28$, main effect of age; $F_{(1,14)}=0.76$, $P=0.39)$. However, there was a main effect of pattern $\left(F_{(2,28)}=\right.$ 4.36, $P<0.05)$. Post hoc testing did not show any differences in age-related performance between groups. The second reversal did not show any statistically significant effect on latency (interaction effect; $F_{(2,28)}=0.39, P=0.68$, main effect of age; $F_{(1,14)}=$ 0.03, $P=0.88$, main effect of pattern; $F_{(2,28)}=0.97, P=0.39$, Fig. $3 \mathrm{C})$. In addition, we observed that the average latency decreased from task 1 to task 2-this is likely due to a practice effect or because as the animals learn the idea that they get rewarded on responding, the response rate becomes faster. Overall, the data suggest that this task may be insufficiently challenging and/or the animals were using alternative strategies such as (1) an allocentric strategy by using the corners as an external reference points to reach the correct response, (2) learning only the correct location, or (3) using an egocentric strategy by choosing correct object based on reference to self. Hence we modified the test to increase task complexity and to tease out the relevant strategy used by the dogs.

\section{Six-well pattern discrimination test}

In a modified version of the above described four-well task, we used a six-well pattern discrimination task where the position of both the correct and "incorrect" objects were varied and could be to the right or left of the animal. Specifically, animals were presented with repeated sequence of spatial pairs, in sequences of three-that is, spatial pattern 1 (Fig. 4B), spatial pattern 2 (Fig. $4 \mathrm{C}$ ), and spatial pattern 3 (Fig. 4D) repeated over the 45 trials. This six-well task was designed to be a more complex version of the four-well task to tease out age differences. We designed the task such that there would be a linear decline in performance as a function of increasing interference from spatial pattern 1-2-3. We hypothesized that age differences would become more evident as interference was increased (i.e., a group by condition effect). The results, using a two-way ANOVA, showed that aged animals performed more poorly than young animals $\left(F_{(1,13)}=\right.$ 14.38, $P<0.01)$. Further post hoc testing demonstrated that this 


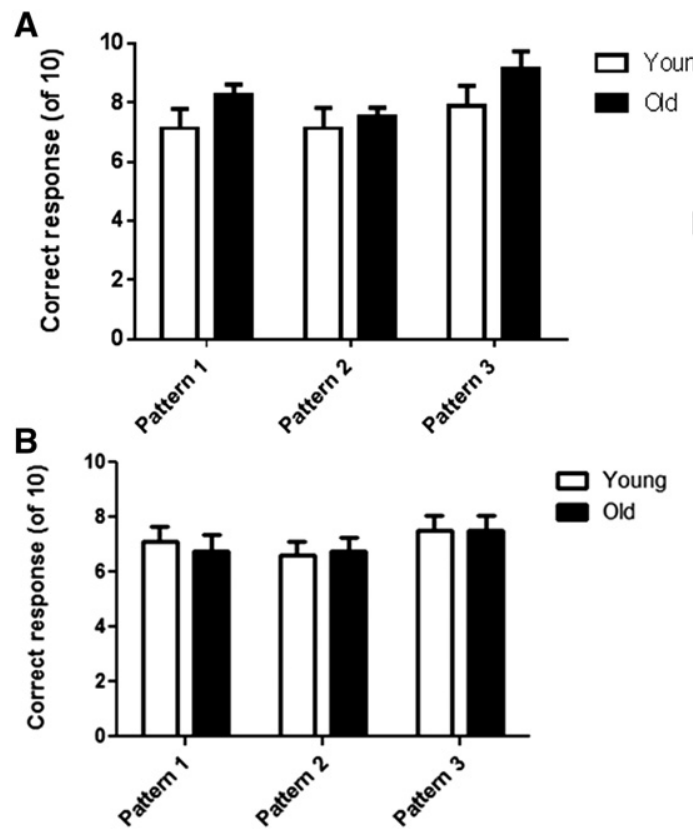

Learning

Reversal 1

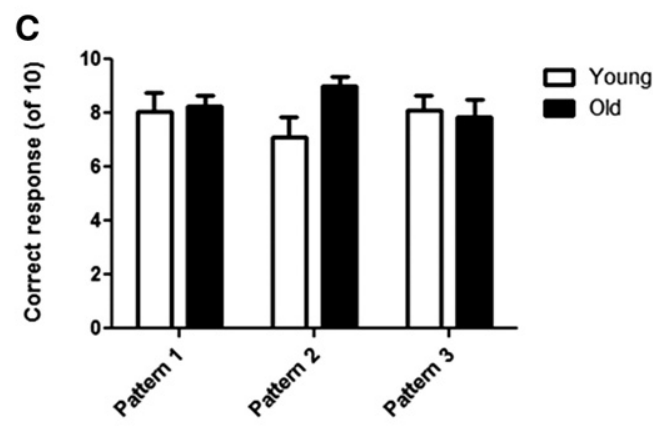

Reversal 2

Figure 2. Performance of young and old animals on the four-well task showed no significant between-group differences. ( $A$ ) Correct responses recorded on the initial learning phase of the task. $(B)$ Correct responses recorded on the first reversal phase of the task. (C) Correct responses recorded on the second reversal phase of the task errors. Error bars represent standard errors.

effect was selective for pattern $2(P<0.01)$ and pattern $3(P<$ 0.05 ) but not for problem 1 (Fig. 5A). We also found a significant effect of task $\left(F_{(2,26)}=14.59, P<0.01\right)$. However, there was no overall interaction effect $\left(F_{(2,26)}=1.48, P=0.24\right)$.

We did not observe any significant effect on latency (interaction effect; $F_{(2,18)}=0.41, P=0.66$, main effect of age; $F_{(1,9)}=1.57$, $P=0.24$, Fig. 6A). It is important to note that there was a trend for task effect, but this did not reach significance (main effect of task $\left.\left(F_{(2,18)}=3.22, P=0.06\right)\right)$.

As expected, reversal learning was more difficult than original learning and all animals made more errors on the reversal phase than on the previous learning phase (confirmed by a twoway ANOVA that showed significant main effect of test phase $\left(F_{(1,75)}=8.75, P<0.01\right)$ and of pattern $\left(F_{(2,75)}=6.68, P<0.01\right)$, multiple comparison corrected $t$-tests showed significant differences on pattern 1 and pattern $3(P<0.05)$ and a trend for pattern $2(P=0.06)$, Fig. 5B). However, we had also hypothesized that reversal learning will occur more rapidly in young animals than old animals. A two-way ANOVA supported this hypothesis. There was a significant effect of both age $\left(F_{(1,10)}=16.49, P<0.01\right.$, Fig. 5B) and task $\left(F_{(1,10)}=6.28, P<0.01\right)$ and only a marginal interaction effect $\left(F_{(1,13)}=3.20, P=0.06\right)$. Further post hoc analysis demonstrated that aged animals performed worse on pattern 2 compared with young animals $(P<0.01)$. This effect was absent on both pattern 1 and pattern 3 indicating that animals were potentially using a pattern separation strategy in patterns 1 and 2 but in pattern 3 the animals had either (1) learnt that the principle was now win-stay, lose-shift and used that strategy successfully or (2) were showing perseverative responding for the object that was located on the side that was rewarded more often during the training trial (Fig. 4A), spatial pattern 1 (Fig. 4B), and spatial pattern 3 (Fig. 4D) .

Finally, we had hypothesized that reversal 2 learning will occur more rapidly than reversal 1 learning since the animals were now well trained on the task and reaching "criterion" quicker
(Fig. 5C). Our data confirm this hypothesis, with all animals acquiring this task faster than the learning or reversal 1. Finally as with the first reversal, there was a significant effect of age $\left(F_{(1,10)}=6.31\right.$, $P<0.05$, Fig. 5B) but not task $\left(F_{(2,18)}=2.62, P=0.10\right)$ and no interaction effect $\left(F_{(2,18)}=1.81, P=0.19\right)$. Further post hoc analysis demonstrated that aged animals performed worse on problem 2 compared with young animals $(P<0.05)$.

As with the other problems in the task, we did not observe any significant effect on latency (interaction effect; $F_{(2,20)}=$ $0.97, P=0.39$, main effect of age; $F_{(1,10)}=0.67, P=0.43$, Fig. $6 \mathrm{~A}$, main effect of task $\left(F_{(2,20)}=0.92, P=0.41\right)$.

\section{Six-well pattern separation test}

In order to evaluate whether pattern separation deficits influenced performance of young versus old dogs and exclude other potential explanations, we tested the animals on another version of the six-well pattern separation task involving two problem sets. The order of task presentation was varied so that problem set 1 could either be presented first or second This task also included two reversals (one in going from problem sets 1-2) and again within problem 2 where the rewarded object was moved from left to right. This was done in order to determine whether aged animals show more perseveration, that is, continue to go to the previously correct side despite the change of stimuli and to tease apart potential contributions of prefrontal impairment.

Our data show that on problem set 1 , where the correct object is always to the left-old and young animals perform equally well (interaction effect; $F_{(2,20)}=1.59, P=0.27$, main effect of age; $\left.F_{(1,10)}=0.60, P=0.45\right)$, although the task did get progressively harder for all animals $\left(F_{(2,20)}=26.922, P<0.01\right.$, Fig. 7B). We did not observe any significant effect on latency either although there was an interaction effect (interaction effect; $F_{(2,20)}=11.17$, $P<0.01$, main effect of age; $F_{(1,10)}=0.73, P=0.49$, main pattern effect $\left.\left(F_{(2,20)}=0.40, P=0.60\right)\right)$. This confirmed the hypothesis 


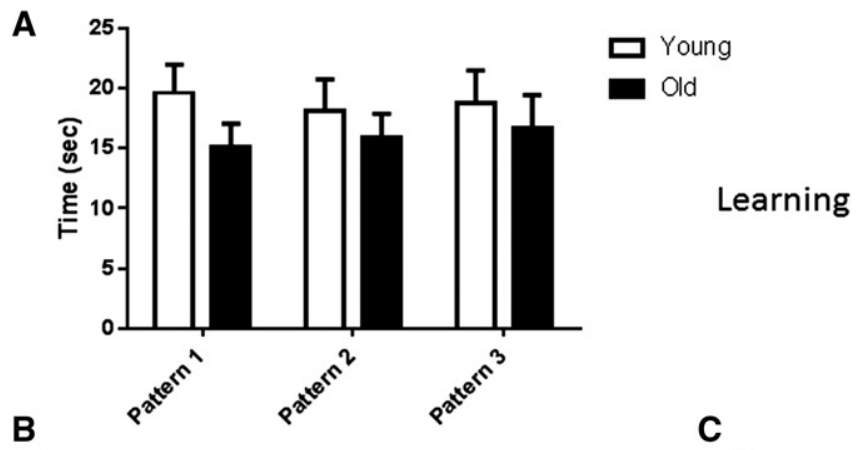

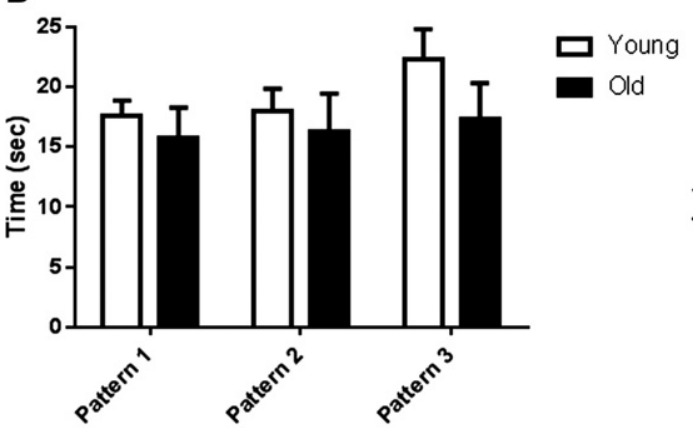

Reversal 1



Reversal 2

Figure 3. Latency measures of young and old animals on the four-well task showed no significant between-group differences. ( $A$ ) Response latency recorded on the initial learning phase of the task. (B) Response latency recorded on the first reversal phase of the task. (C) Response latency recorded on the second reversal phase of the task errors.

(as seen in Fig. 7A) that both aged and young dogs are able to perform equally on a task when the objects are always to one side even if they are brought closer.

In contrast, there was a significant effect of age on problem set 2 (Fig. 8A), where the corners were still used but the correct choice could be either toward the left or toward the right (interaction effect; $F_{(2,20)}=2.5, P=0.08$, main effect of age; $F_{(1,10)}=5.74, P=0.03$, Fig. $8 \mathrm{~B}$ (despite one young dog performing very poorly and one old dog performing exceedingly well), and a main effect of task $\left(F_{(2,20)}=8.63, P<0.01\right)$ consistent with problem set 1 . We did not observe any significant age-related effect on latency on this problem set either although there was a pattern effect (interaction effect; $F_{(2,20)}=0.51, P=0.61$, main effect of age; $F_{(1,10)}=0.06, P=0.82$, main pattern effect $\left(F_{(2,20)}=4.60, P<0.05\right.$, Fig. 8C)).

As opposed to the pattern discrimination test where the previously rewarded object becomes the location for the nonrewarded object (Fig. 4C), in this test the right and left sides were switched but different locations were used (Fig. 8A) thus more clearly delineating pattern separation from reversal learning. Our results on this task, are consistent with previous pattern separation tests in humans and rodents and show age-related
A

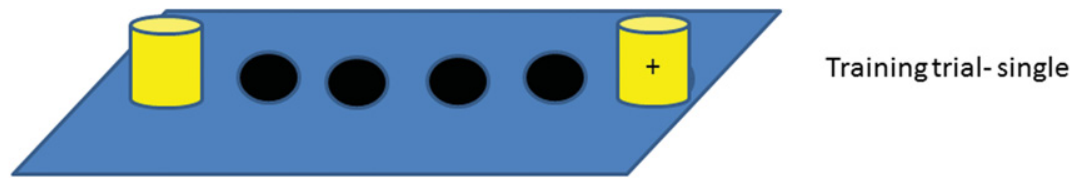

B

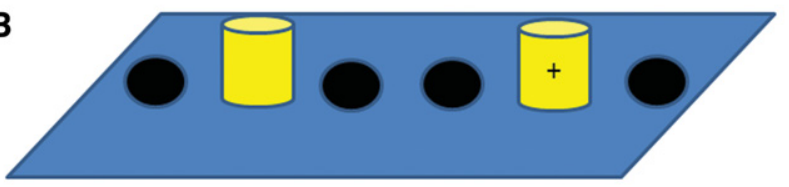

Spatial Pattern 1

C

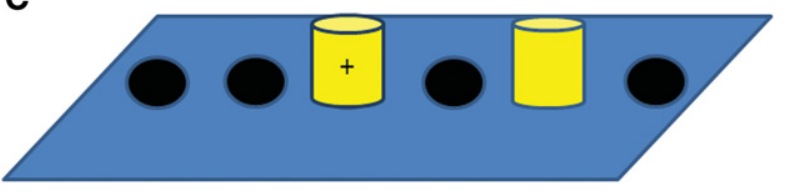

Spatial Pattern 2

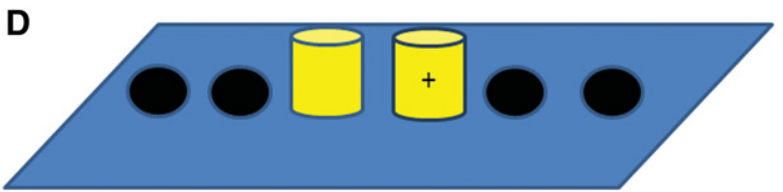

Spatial Pattern 3

Figure 4. Spatial configuration of objects presented to the animals during the six-well pattern discrimination task. Pattern 1 is considered easiest with the distance between objects being the greatest and pattern 2 and pattern 3 having progressively lesser distance between objects. $(A)$ Animals were given one training trial where they were rewarded for choosing either object as shown in the figure. This was done to ensure that the animals identified corners as possible options. (B) Animals were presented with the pairs of objects as shown in the figure during the learning phase of the task. (C) Animals were presented with the pairs of objects as shown in the figure during the first reversal phase of the task. (D) Animals were presented with the pairs of objects as shown in the figure during the second reversal phase of the task. 
A

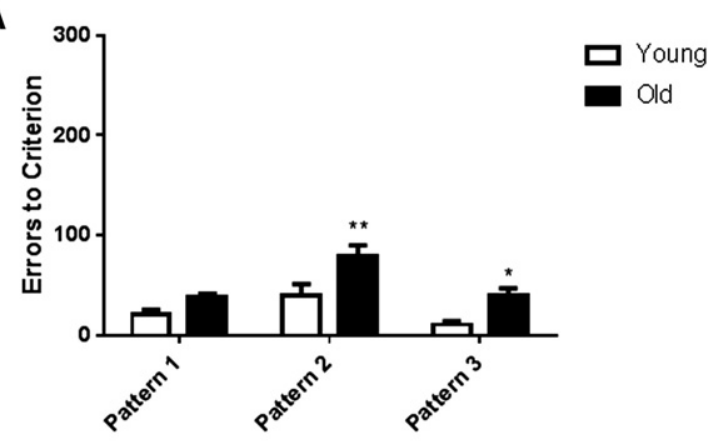

Learning

B

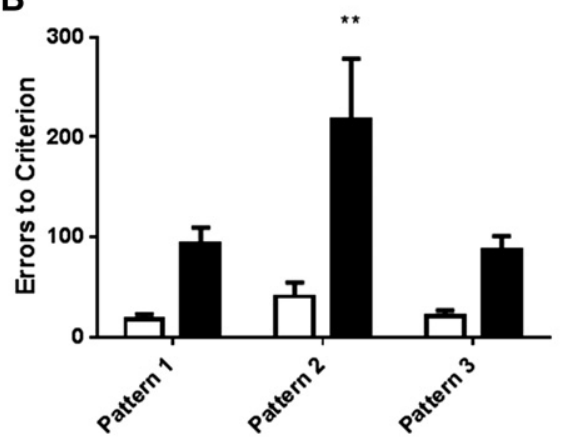

Reversal 1

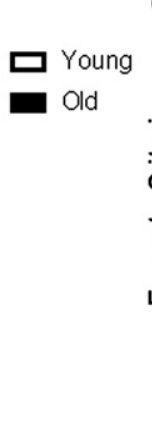

C

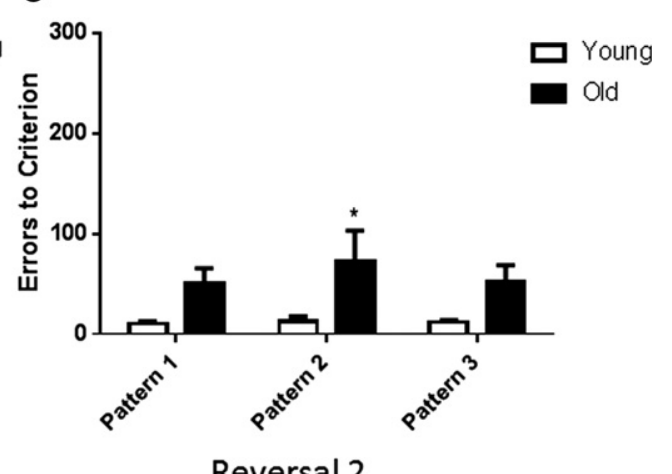

Figure 5. Performance of young and old animals on the six-well pattern discrimination task showed significant age-related differences between groups. (A) Errors to criterion recorded on the initial learning phase of the task showed significant differences between young and old animals on patterns 2 and 3. (B) Errors to criterion recorded on the first reversal phase of the task showed significant differences between young and old animals on pattern 2 alone. (C) Errors to criterion recorded on the second reversal phase of the task errors showed no significant between-group differences. Error bars represent standard errors. $\left(^{* *}\right) P<0.01,\left({ }^{*}\right) P<0.05$ significant difference in performance compared with young controls, Bonferroni test.

difference in performance on the task, with a better performance on Pattern 1 (wide separation) than Pattern 3 (small separation) for all animals and significant differences between aged versus young animals only when there is a small separation between objects.

Overall these results suggest that the existence of age-related differences depends at least in part on the difficulty of the task, and that aged dogs are not compromised in their ability to learn simple pattern separation tasks. However, as the task complexity is increased, their performance declines compared with younger animals. In addition, the comparison of problem set 1 versus problem set 2 clearly indicates an impairment in older canines on the more difficult condition, when it involves a reversal learning component (Fig. 5A,B) but also when there is a pattern separation component alone (Fig. 8B).

\section{Discussion}

This study was designed to evaluate spatial discrimination abilities (thought to index spatial pattern separation) as a function of age in the aged dogs. There were two main hypotheses; (1) difficulty would vary linearly with the distance between the two stimuli; the further the distance, the less the interference and thus, the easier the problem from a pattern separation perspective; (2) age difference(s) on the tasks would be most apparent on the more complex problems compared with the simpler problems. Our results demonstrate that while age differences are observable on more complex tasks (six-well task) relative to easier tasks (fourwell task), the age-related deficits maybe due to loss of perceptual and perhaps inhibitory control if the location of one or the other object remains same. Pattern separation is tested when the task is modified so that objects are brought closer together, with different locations used for each object. The age-related differences in pattern separation emerge clearly in such a case even when the correct object is switched from left to right. This work builds on and extends previous findings which demonstrate that pattern separation relies on neural networks that extend beyond the hippocampus.

In a six-well task that directly assessed pattern separation, we found that aged animals were impaired on the more challenging spatial arrangement of the task (Fig. 8A) where the objects are closer together while also switching the sides from left to right. While the aged animals made significantly more errors in choosing the correct object when the objects were close together, there was no difference in the latency of response between any of the groups suggesting that the difference in performance stems from a "pattern separation deficits" rather than speed of processing. This finding is consistent with and significant in the context of human and rodent literature on pattern separation where age is an important predictor of performance (Toner et al. 2009; Stark et al. 2010). However, our findings also suggest that modified versions of this task can be used to identify age-related deficits in other processes (such as context switching and inhibitory response) that are affected by age. Specifically in another version of the sixwell task described above, (referred to as the pattern discrimination task Fig. 4A-D), we found that aged animals were impaired on patterns 2 (intermediate spatial distance between objects) and pattern 3 (small spatial distance between objects) but not on pattern 1 (large distance between objects). These results support the hypothesis that pattern discrimination abilities decline with age as the distances between objects gets closer. However, 


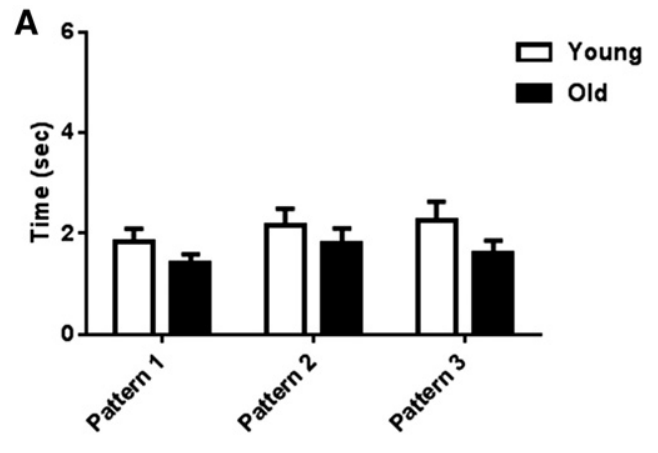

\section{Learning}

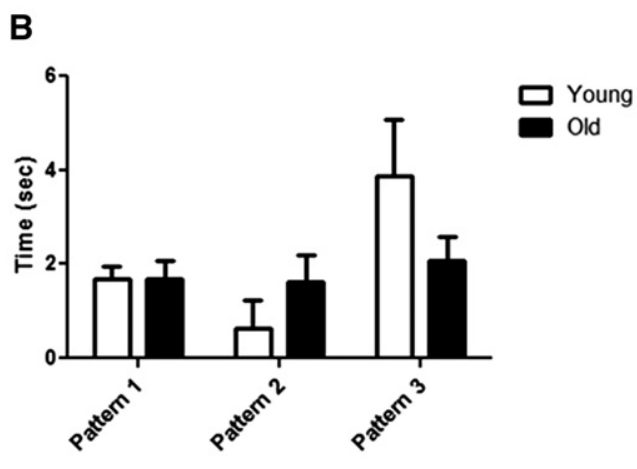

Reversal 1

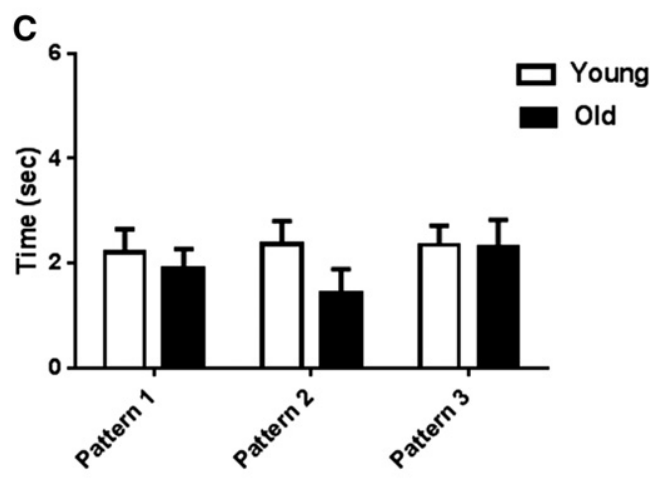

Reversal 2

Figure 6. Latency measures of young and old animals on the six-well pattern discrimination task showed no age-related differences between groups. $(A)$ Response latency recorded on the initial learning phase of the task. (B) Response latency recorded on the first reversal phase of the task. (C) Response latency recorded on the second reversal phase of the task errors.

\section{A}

Problem set 1

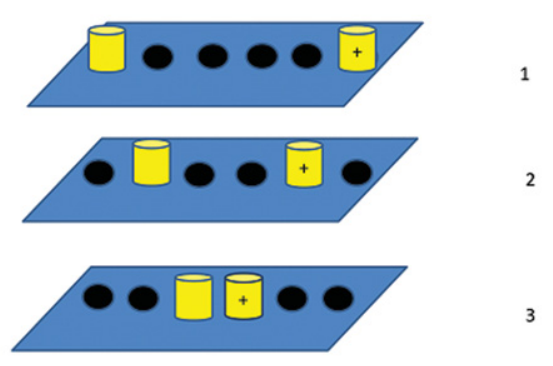

B

Problem set 1 (Days 1-6)

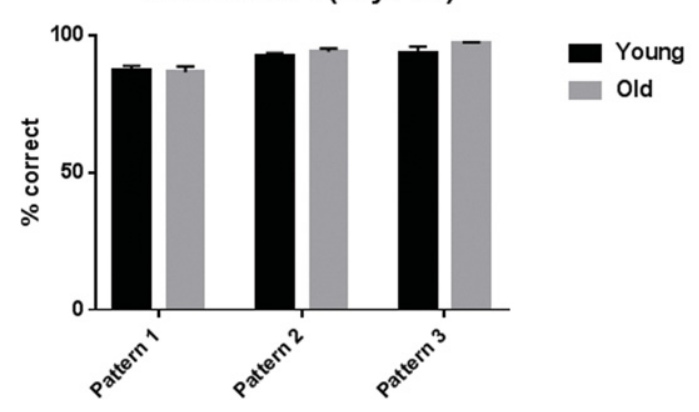

C

Problem set 1 (Days 1-6)

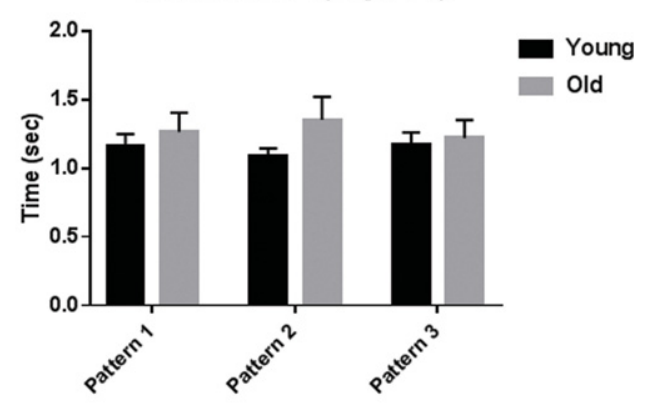

Figure 7. (A) Spatial configuration of objects presented to the animals during problem set 1 in the six-well pattern separation task. Neither the $(B)$ percentage of correct response recorded on the task over six sessions for each problem set (over $6 \mathrm{~d}$ ) nor (C) response latency recorded on the task showed any significant between-group differences. Error bars represent standard errors. 


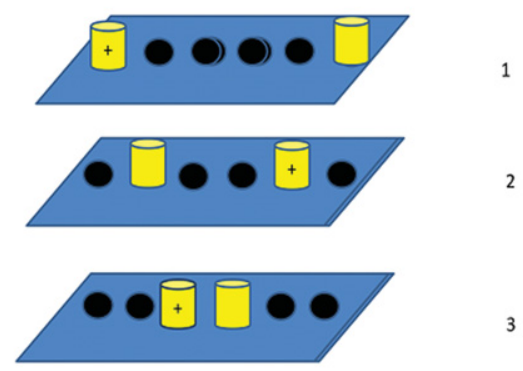

\section{B}



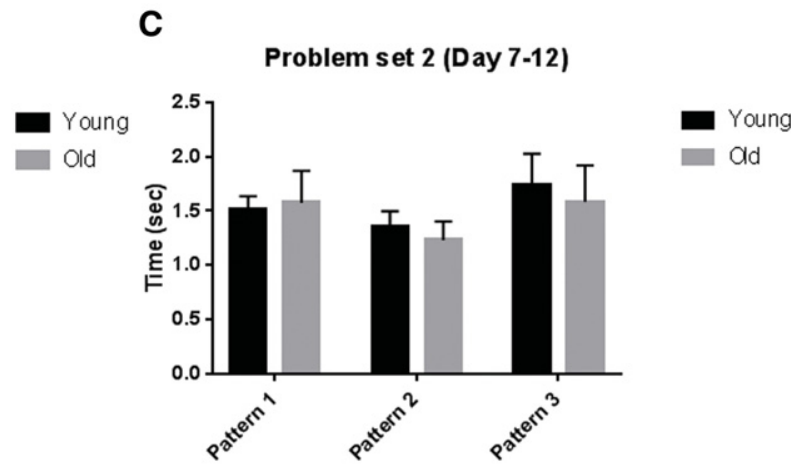

Figure 8. (A) Spatial configuration of objects presented to the animals during problem set 2 on the six-well pattern separation task. There was a significant effect of age on problem set 2 as seen in the $(B)$ percentage of correct responses recorded on the task measured over six sessions for each problem set (over $6 \mathrm{~d}$ ), but not on the $(C)$ response latency recorded on the task. Error bars represent standard errors. $\left(^{*}\right) P<0.05$ significant difference in performance compared with young controls, Bonferroni test.

the intriguing finding from this study is that all animals found pattern 2 harder to learn than pattern 3. Pattern 2 required animals to recognize that the correct object was now located on the left rather than the right side, and that what was previously the rewarded location, had now become the nonrewarded location in addition to the reduction in distance between the objects compared with pattern 1 . On pattern 3 , the correct object was once again returned to the right of the animal (as in pattern 1 , and once again what was previously the rewarded location, had now become the nonrewarded location) but the distance between correct and incorrect object was even closer. If the animals had relied solely on spatial distance in responding, we would have observed more errors on pattern 3 as has been observed previously in aged dogs (Milgram et al. 2002). However, it appears that aged animals used either (a) an egocentric strategy (b) or demonstrated perseverative responding to a given side demonstrating an inability to shift strategy in a goal-directed manner. In order to examine this further, we continued the task to test reversal learning on the same task.

Consistent with our hypothesis, in the first reversal phase of the task, all animals (young and old) made more errors than on the previous learning phase of the task. However, aged animals once again showed significant increase in errors on pattern 2, demonstrating their inability to shift strategy when the goals shift demonstrating impaired executive function but not learning compared with young animals. Finally, as seen in Figure 5C on the reversal 2 phase of this task, learning occurred much more rapidly than reversal, suggesting that this very rapid learning reflected the acquisition of a new strategy, that of win-stay, lose-switch, which is likely to be the result of extensive training on the task for both young and old animals. It should also be noted, that the use of reversal strategy on a pattern separation task has not been described in the past and sheds some light on the executive function components of this task.

In contrast to the six-well task, the four-well task revealed no age-related differences in performance. This highlights the relevance of task complexity to tease out age differences on cognitive function. It is likely that simple learning and simple spatial discrimination ability is not impaired in aging dogs. We found no difference between young and old dogs in latency to respond in either the six-well or the four-well tasks suggesting that speed of processing did not impact the outcome measures for these tests.

Overall, these results suggest that aging in dogs is associated with the loss of selective processes of pattern separation and executive function but not of simple spatial pattern discrimination processes or speed of processing. This is consistent with previous work, which has demonstrated that age-related declines in pattern separation are facilitated by decline in limbic tract integrity, and cortical disconnection (Bartzokis 2004; O'Sullivan et al. 2001). While the comparison of pattern 1 versus pattern 2 in the pattern discrimination task and problem set 2 versus problem set 1 in the pattern separation task clearly indicate a disproportionate impairment in older canines on the more difficult condition, consistent with a hippocampal pattern separation deficit (Neunuebel and Knierim 2014; Yassa et al. 2011a), our results also show that canines are capable of devising alternate strategies in order to solve simpler spatial pattern problems.

This work contributes to a growing body of work on agerelated memory impairments in pattern separation and demonstrates that cognitive deficit in aging may be higher order perceptual rather than spatial learning processing. 


\section{Materials and Methods}

\section{Subjects}

Initially, a total of eight young beagles (1-5 yr of age, including males and females) and eight aged beagles ( $>7 \mathrm{yr}$ of age, including males and females) from CanCog Technologies Inc. (Toronto, Canada) were used as subjects. Age-related cognitive deficits are known to start as early as $6 \mathrm{yr}$ of age in beagle dogs (Studzinski et al. 2006), which is the basis for the age range chosen for this study. At the start of the study, all dogs had similar cognitive backgrounds, which included completing a standard program of pretraining, as described previously (Milgram et al. 1994) and also pretraining on a delayed nonmatching-to-position task (Chan et al. 2002). Veterinary examinations conducted on all dogs prior to the start of the study established that all subjects were in good general health, with uncompromised visual, auditory, and motor functioning. Experimenters were not blinded to the ages of the dogs. Most testers work with the animals on a daily basis and at minimum know the age range of every dog.

\section{Housing}

Dogs were group housed based on compatibility up to four per pen, in five by $16.5 \mathrm{ft}$ pens and had free access to water for the duration of the study. They were fed a standard adult maintenance diet of (chicken and rice) so as to maintain a consistent body weight. Housing temperature and humidity were maintained by automated temperature control and continuous ventilation. Room environmental conditions have design specifications as follows: single-pass air supply with 2200 c.f. filtered air changes per minute, relative humidity of $60 \% \pm 10 \%$, temperature of $21^{\circ} \mathrm{C} \pm 3^{\circ} \mathrm{C}$, and a natural light-dark cycle. The study protocol was approved by the CanCog Technologies Institutional Animal Care Committee, and it followed the guidelines of the Ontario Ministry of Agriculture.

\section{Behavioral testing apparatus}

All cognitive testing was conducted in a modified canine adaptation of the Wisconsin general testing apparatus, as originally described by Milgram et al. (1994). Briefly, the apparatus consisted of a holding area where the dog was housed during testing, which was separated from the experimenter by a plastic screen containing a one-way mirror and a hinged door at the bottom. A Plexiglas stimulus tray (22-in long and 10-in deep) containing four or six equally spaced food wells was pushed through the hinged door by the experimenter so that the dog could access the stimuli (red lego blocks, 3 in $\times 3$ in $\times 3$ in in size) and food rewards by sticking its head through adjustable stainless steel gates at the front of the holding area. The tray was moved out of sight during delay and inter-trial intervals.

Food reward for correct responses during cognitive testing consisted of $\sim 1 \mathrm{~g}$ of wet $\operatorname{dog}$ food (Hill's P/D). To mask the presence of the food reward in the positive food wells, the undersides of all stimuli were baited with the same food such that, while able to smell it, the animals could not see or eat it.

\section{Cognitive testing}

This behavioral protocol on which the animals were tested was intended to provide a means of assessing pattern separation in beagle dogs and is based on the assumption that performance on a spatial discrimination learning task is accomplished by hippocampal neural circuitry that can represent distinct stimulus patterns. The testing procedure examined performance of beagle dogs on a novel protocol that assessed learning on three concurrent spatial discrimination tasks, with each task requiring the subject to select one of two identical objects of an object pair based on spatial position. The three spatial configurations used overlapping positions in which the distance between the objects varied from large or wide $(\sim 15 \mathrm{in})$, to medium $(\sim 10 \mathrm{in})$, and to low ( $\sim 5 \mathrm{in})$, with task difficulty hypothesized to vary inversely with spatial distance. The same animals were used for the first two tasks and to minimize any residual effects between tasks-the tasks were conducted more than 1-mo apart. The third task was conducted on a new group of animals.

\section{Four-well pattern separation test}

We first examined performance using a four-location target field in which the spatial pairs were presented in 10 consecutive blocks of three problems per block. Thus, each problem was presented on 10 occasions per day. There was a 2-sec inspection interval, which allowed the dog to examine both objects before the tray was fully extended for the animal to make a response, and the inter-trial interval was $15 \mathrm{sec}$. On the initial learning, the correct responses were as indicated in Figure 1, which assured that no position is correct for more than one problem. In addition, in this initial task, the location of the incorrect response was maintained constant.

Subjects were tested until they reached $70 \%$ correct. However, in this test all animals reached criterion in $1 \mathrm{~d}$, so performance is measured as "number of correct response" out of a total of 10. After completing the first phase (learning, Fig. 1A), the location of each correct object was reversed (Fig. 1B) and the testing procedure described above was repeated. After completing the first reversal, the positions were reversed again (Fig. 1C) and subjects were given a second reversal trial.

\section{Six-well pattern discrimination test}

Training trial. Prior to start of the task, animals were given a single training trial with one object in a corner being rewarded (Fig. 4A). This was done to ensure that animals considered the corners as possible options, although for further testing corners were not used (Fig. 4).

Test trials. Testing entailed presenting repeated sequence of spatial pairs with 45 pairs presented per day, in sequences of three-that is, problem 1, 15 trials; problem 2, 15-trials; problem 3 repeated over the 45 trials (Fig. 4B-D). The inter-trial interval was $15 \mathrm{sec}$ and there was a 2 -sec inspection interval before presenting the tray to the dog. On the initial learning, the correct responses was as indicated in Figure 4, which intentionally assures that no position is correct for more than one problem. Subjects were tested until they reach $70 \%$ correct over the entire 45 trials day before moving to the reversal phase 1 (where the location of each correct object was reversed) and then reversal phase 2 (where the correct object was the same as in the learning phase) once they reached criterion for reversal phase 1.

\section{Six-well pattern separation test}

In another modification of the six-well task, animals were presented with repeated sequence of spatial pairs with each session containing 45 trials ( 15 per problem) and each subject received one session per test day. As the order of problem could be randomized in six ways, the dogs received six sessions for each problem set (over $6 \mathrm{~d}$ ).

Problem set 1 . Problem set 1 consists of three problems assessing spatial discrimination learning, using distinct locations, with no location used more than once. In problem set 1 , all three correct locations are located to the animals left in sequences of threethat is, problem 1 , problem 2 , and problem 3 were repeated over the 45 trials and number of correct response over this fixed number of trials was recorded (Fig. 7). This six-well task was designed to be more complex version of the four-well task to tease out age differences.

Problem set 2. Immediately after the first $6 \mathrm{~d}$, all dogs were tested on problem set 2 (Fig. 8A). Problem set 2 used the same locations as problem set 1, but two of the three are located on the right of the animal. The number of correct responses over 45 trials as described above (problem set 1) was recorded. Thus, this test 
measured a context switch from left to right and also teased out if it was the context switch or distance between objects that would impact performance.

\section{Statistics}

All statistical analyses were conducted using GraphPad Prism. Repeated-measures analysis of variance (ANOVA) or one-way ANOVA was used to assess between-group differences. Bonferroni post hoc $t$-test was conducted to examine group differences when a significant overall effect was found. Where a main effect was found but no interaction in the ANOVA, $t$-tests were conducted a priori.

\section{Acknowledgments}

This work was funded in part by the grants AG012694-16 and PPG AG000538 from the National Institute of Aging. Support for M.A.Y. was provided by R01 MH102392, R21 AG049220, and P50 AG16573.

\section{References}

Aimone JB, Deng W, Gage FH. 2011. Resolving new memories: a critical look at the dentate gyrus, adult neurogenesis, and pattern separation. Neuron 70: $589-596$.

Bakker A, Krauss GL, Albert MS, Speck CL, Jones LR, Stark CE, Yassa MA, Bassett SS, Shelton AL, Gallagher M. 2012. Reduction of hippocampal hyperactivity improves cognition in amnestic mild cognitive impairment. Neuron 74: 467-474.

Bartzokis G. 2004. Age-related myelin breakdown: a developmental model of cognitive decline and Alzheimer's disease. Neurobiol Aging 25: 5-18; author reply $49-62$.

Bennett IJ, Huffman DJ, Stark CE. 2015. Limbic tract integrity contributes to pattern separation performance across the lifespan. Cereb Cortex 25: $2988-2999$.

Bussey TJ, Saksida LM, Rothblat LA. 2001. Discrimination of computer-graphic stimuli by mice: a method for the behavioral characterization of transgenic and gene-knockout models. Behav Neurosci 115: 957-960.

Chan AD, Nippak PM, Murphey H, Ikeda-Douglas CJ, Muggenburg B, Head E, Cotman CW, Milgram NW. 2002. Visuospatial impairments in aged canines (Canis familiaris): the role of cognitive-behavioral flexibility. Behav Neurosci 116: 443-454.

Cotman CW, Head E. 2008. The canine (dog) model of human aging and disease: dietary, environmental and immunotherapy approaches. J Alzheimers Dis 15: 685-707.

Goodrich-Hunsaker NJ, Hunsaker MR, Kesner RP. 2008. The interactions and dissociations of the dorsal hippocampus subregions: how the dentate gyrus, CA3, and CA1 process spatial information. Behav Neurosci 122: 16-26.

Head E, Rofina J, Zicker S. 2008. Oxidative stress, aging, and central nervous system disease in the canine model of human brain aging. Vet Clin North Am Small Anim Pract 38: 167-178, vi.

Marrone DF, Adams AA, Satvat E. 2011. Increased pattern separation in the aged fascia dentata. Neurobiol Aging 32: 2317.e2323-2317.e2332.

Milgram NW, Head E, Weiner E, Thomas E. 1994. Cognitive functions and aging in the dog: acquisition of nonspatial visual tasks. Behav Neurosci 108: $57-68$.

Milgram NW, Head E, Muggenburg B, Holowachuk D, Murphey H, Estrada J, Ikeda-Douglas CJ, Zicker SC, Cotman CW. 2002. Landmark discrimination learning in the dog: effects of age, an antioxidant fortified food, and cognitive strategy. Neurosci Biobehav Rev 26: 679-695.

Neunuebel JP, Knierim JJ. 2014. CA3 retrieves coherent representations from degraded input: direct evidence for CA3 pattern completion and dentate gyrus pattern separation. Neuron 81: 416-427.

O'Sullivan M, Jones DK, Summers PE, Morris RG, Williams SC, Markus HS. 2001. Evidence for cortical "disconnection" as a mechanism of age-related cognitive decline. Neurology 57: 632-638.

Reagh ZM, Roberts JM, Ly M, DiProspero N, Murray E, Yassa MA. 2014. Spatial discrimination deficits as a function of mnemonic interference in aged adults with and without memory impairment. Hippocampus 24: 303-314.

Rich EL, Shapiro M. 2009. Rat prefrontal cortical neurons selectively code strategy switches. J Neurosci 29: 7208-7219.

Rolls ET. 2015. Pattern separation, completion, and categorisation in the hippocampus and neocortex. Neurobiol Learn Mem 129: 4-28.

Stark SM, Yassa MA, Stark CE. 2010. Individual differences in spatial pattern separation performance associated with healthy aging in humans. Learn Mem 17: 284-288.

Studzinski CM, Christie LA, Araujo JA, Burnham WM, Head E, Cotman CW, Milgram NW. 2006. Visuospatial function in the beagle dog: an early marker of cognitive decline in a model of human aging and dementia. Neurobiol Learn Mem 86: 197-204.

Toner CK, Pirogovsky E, Kirwan CB, Gilbert PE. 2009. Visual object pattern separation deficits in nondemented older adults. Learn Mem 16: $338-342$.

Yassa MA, Stark CE. 2011. Pattern separation in the hippocampus. Trends Neurosci 34: 515-525.

Yassa MA, Lacy JW, Stark SM, Albert MS, Gallagher M, Stark CE. 2011a Pattern separation deficits associated with increased hippocampal CA3 and dentate gyrus activity in nondemented older adults. Hippocampus 21: $968-979$.

Yassa MA, Mattfeld AT, Stark SM, Stark CE. 2011b. Age-related memory deficits linked to circuit-specific disruptions in the hippocampus. Proc Natl Acad Sci 108: 8873-8878.

Received June 30, 2016; accepted in revised form December 13, 2016. 


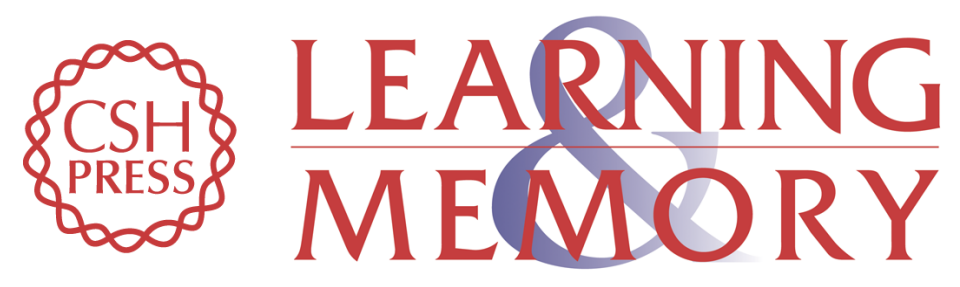

\section{Pattern separation and goal-directed behavior in the aged canine}

Shikha Snigdha, Michael A. Yassa, Christina deRivera, et al.

Learn. Mem. 2017, 24:

Access the most recent version at doi:10.1101//m.043422.116

References This article cites 23 articles, 4 of which can be accessed free at: http://learnmem.cshlp.org/content/24/3/123.full.html\#ref-list-1

Creative This article is distributed exclusively by Cold Spring Harbor Laboratory Press for the Commons first 12 months after the full-issue publication date (see

License http://learnmem.cshlp.org/site/misc/terms.xhtml). After 12 months, it is available under a Creative Commons License (Attribution-NonCommercial 4.0 International), as described at http://creativecommons.org/licenses/by-nc/4.0/.

Email Alerting Receive free email alerts when new articles cite this article - sign up in the box at the Service top right corner of the article or click here. 